\title{
AN OPERATOR THEORETICAL CHARACTERIZATION OF $\varepsilon$-ENTROPY IN GAUSSIAN PROCESSES
}

\author{
Dedicated to Professor Hisaharu Umegaki on his sixtieth birthday
}

\author{
By SHigeo AKASHI
}

\section{Introduction.}

In this paper, we shall treat $\varepsilon$-entropy of compact operators on a Hilbert space and that of measurable stochastic processes. Especially, using the concept of $\varepsilon$-entropy, we study a relation between Gaussian processes and integral kernel operators. In section 2 , we shall explain the definition of $\varepsilon$-entropy in compact operators due to Prosser [9] and that in measurable stochastic processes due to Kolmogorov [5]. In section 3, we shall treat a mean continuous Gaussian process $\xi=\{\xi(t): 0 \leqq t \leqq 1\}$. Using the covariance function $K(s, t)$ induced by $\xi$, we can construct the integral kernel operator $T$ on $L^{2}[0,1]$, which is a trace class operator. Denote $S(T, \varepsilon)$ and $H(\xi, \varepsilon)$ the $\varepsilon$-entropies of $T$ and $\xi$, respectively. We characterize the $\varepsilon$-entropy $H(\xi, \varepsilon)$ by the sequence:

$$
\left\{S\left(T^{k}, \varepsilon^{k}\right): k=1,2, \cdots\right\} .
$$

In section 4, we shall consider the orders of growth of $H(\xi, \varepsilon)$ and $S(T, \varepsilon)$. Then, applying the result of Section 3, we estimate an upper bound of the order of growth of $H(\xi, \varepsilon)$.

Unless stated otherwise, throughout this paper, the letters $R, Z$ and $N$ denote the set of real numbers, the set of integers and the set of natural numbers, respectively.

\section{Preliminaries.}

In this section, we shall introduce several notations and definitions throughout this paper. Denote by $\mathscr{H}$ a Hilbert space whose inner product is $\langle\cdot, \cdot\rangle$. $B(x, \varepsilon)$ means an open ball having the radius $\varepsilon>0$ and the center $x \in \mathscr{H}$. Especially, denote by $\mathcal{U}$ the closed unit sphere in $\mathscr{H}$.

By an $\varepsilon$-covering of a subset $F$ in $\mathscr{H}$, we mean a family of open balls with centers in $\mathscr{H}$ and radiuses $\varepsilon$, whose union covers $F$. By an $\varepsilon$-packing of $F$, we mean a family of open balls with centers in $F$ and radiuses $\varepsilon$, whose pairwise

Received March 19, 1985 
intersections are all empty. If $F$ is relatively compact, there are finite $\varepsilon$ coverings and $\varepsilon$-packings of $F$ for every $\varepsilon>0$. Denote by $N(F, \varepsilon)$ the minimum number of balls in any $\varepsilon$-covering of $F$ and by $M(F, \varepsilon)$ the maximum number of balls in any $\varepsilon$-packing of $F$. According to Prosser [9], we define the $\varepsilon$ entropy $S(F, \varepsilon)$ of $F$ as the base-2 logarithm of $N(F, \varepsilon)$ and define the $\varepsilon$-capacity $C(F, \varepsilon)$ of $F$ as the base-2 logarithm of $M(F, \varepsilon)$.

Let $T$ be a compact positive operator on $\mathcal{H}$. When we use the spectral decomposition theorem, the spectral representation of $T$ by using Schatten form (Schatten [10]) is the following:

$$
T=\sum_{i=1}^{\infty} \lambda_{i}\left(\varphi_{i} \otimes \bar{\varphi}_{\imath}\right),
$$

where $\left\{\lambda_{2}\right\}$ is the monotone non-increasing sequence of positive numbers which converges to 0 , and $\left\{\varphi_{i}\right\}$ is the orthonormal system associated with $T$.

For any $r>0$, we introduce a notation $n(r)$ for the number of terms in the sequence $\left\{\lambda_{i}\right\}$ which are greater than $r$ :

$$
n(r)=\max \left\{i: \lambda_{2}>r\right\} \text {. }
$$

For simplicity, we denote by $S(T, \varepsilon)$ the $\varepsilon$-entropy of $T U=\{T x:\|x\| \leqq 1\}$. We call this the e-entropy of $T$.

Let $(X, d)$ be a complete separable metric space and $\mathscr{X}$ be the Borel field generated by the metric $d$. For the random variables $\xi$ and $\eta$ on $(\Omega, \mathscr{B}, P)$ with values in $(X, \mathscr{X})$, let $P_{\xi}, P_{\eta}$ be Borel probability measures on $(X, \mathscr{X})$ induced by $\xi, \eta$, respectively, $P_{\xi, \eta}$ be the joint probability measure of $\xi$ and $\eta$ and $P_{\xi} \times P_{\eta}$ be the product probability measure. The mutual information $I(\xi, \eta)$ between $\xi$ and $\eta$ is defined as follows (Pinsker [8]): If $P_{\xi, \eta} \ll P_{\xi} \times P_{\eta}$,

$$
I(\xi, \eta)=\int_{X \times X} \log \frac{d P_{\xi, \eta}}{d P_{\xi} \times P_{\eta}} d P_{\xi, \eta},
$$

and otherwise, $I(\xi, \eta)=\infty$.

Then the $\varepsilon$-entropy of $\xi$ (Kolmogorov [5]) is defined as

where

$$
H(\xi, \varepsilon)=\inf \left\{I(\xi, \eta): \eta \in W_{\varepsilon}(\xi)\right\}
$$

$$
W_{\varepsilon}(\xi)=\left\{\eta:\left(\int_{\Omega} d(\xi, \eta)^{2} d P\right)^{1 / 2} \leqq \varepsilon\right\}
$$

Let $M$ be the set of all measurable stochastic processes and $\{\xi(t): 0 \leqq t \leqq 1\}$ be a measurable stochastic process defined on $(\Omega, \mathscr{B}, P)$ which satisfies the following inequality :

$$
\|\xi\|_{2}=\left\{\int_{\Omega} \int_{0}^{1}|\xi(t, \omega)|^{2} d t d P(\omega)\right\}^{1 / 2}<\infty
$$

Since we can regard $\xi$ as the random variable from $\Omega$ to $L^{2}[0,1]$ (Baba-KajiIhara [2]), the $\varepsilon$-entropy of $\{\xi(t): 0 \leqq t \leqq 1\}$ is defined as follows : 
where

$$
H(\xi, \varepsilon)=\inf \left\{I(\xi, \eta): \eta \in W_{\varepsilon}(\xi)\right\}
$$

$$
W_{\varepsilon}(\xi)=\left\{\eta \in M:\|\xi-\eta\|_{2} \leqq \varepsilon\right\} .
$$

\section{3. $\varepsilon$-Entropy of Gaussian processess and of integral kernel operators.}

Let $\mathcal{S}$ be a closed subspace of $\mathscr{H}$. We use the following notations :

$$
\begin{gathered}
B_{s}(0, \varepsilon)=B(0, \varepsilon) \cap S, \\
B_{s}(y, \varepsilon)=y+B_{s}(0, \varepsilon), \quad y \in S,
\end{gathered}
$$

that is, $B_{s}(0, \varepsilon)$ is an open $\varepsilon$-ball in $S$ with the center 0 and $B_{s}(y, \varepsilon)$ is an open $\varepsilon$-ball in $\mathcal{S}$ with the center $y \in \mathcal{S}$. Furthermore, let $F$ be a relatively compact subset in $\mathcal{S}$ and denote by $N_{s}(F, \varepsilon)$ the minimum number of balls in any $\varepsilon$-covering of $F$ in $\mathcal{S}$. The $\varepsilon$-entropy $S_{s}(F, \varepsilon)$ of $F$ in $\mathcal{S}$ is defined by

$$
S_{s}(F, \varepsilon)=\log N_{s}(F, \varepsilon),
$$

then we can prove the following lemma:

LEMMA 1.

$$
S(F, \varepsilon)=S_{s}(F, \varepsilon) .
$$

Proof. It is enough to prove that $N(F, \varepsilon)=N_{s}(F, \varepsilon)$. Let $\left\{B\left(x_{\imath}, \varepsilon\right): i=1, \cdots\right.$, $N(F, \varepsilon)\}$ be an $\varepsilon$-covering of $F$ in $\mathcal{H}$, and $\left\{B_{s}\left(y_{\jmath}, \varepsilon\right): j=1, \cdots, N_{s}(F, \varepsilon)\right\}$ be an $\varepsilon$-covering of $F$ in $\mathcal{S}$. As we have $B_{s}\left(y_{\jmath}, \varepsilon\right) \subset B\left(y_{\jmath}, \varepsilon\right)$ for any $j=1, \cdots, N_{s}(F, \varepsilon)$, the family $\left\{B\left(y_{\jmath}, \varepsilon\right): j=1, \cdots, N_{s}(F, \varepsilon)\right\}$ of open $\varepsilon$-balls in $\mathcal{H}$ is also an $\varepsilon$-covering of $F$ in $\mathcal{H}$. From this we have $N(F, \varepsilon) \leqq N_{s}(F, \varepsilon)$.

Let $P$ be the projection from $\mathcal{H}$ to $\mathcal{S}$. Then a family $\left\{B_{s}\left(P x_{\imath}, \varepsilon\right): i=1, \cdots\right.$, $N(F, \varepsilon)\}$ of open $\varepsilon$-balls in $\mathcal{S}$ is also an $\varepsilon$-covering of $F$ in $\mathcal{S}$. In fact, when we choose a fixed $i$ in $\{1, \cdots, N(F, \varepsilon)\}$, we have

$$
\left\|z-P x_{i}\right\|=\left\|P\left(z-x_{\imath}\right)\right\| \leqq\left\|z-x_{i}\right\|<\varepsilon .
$$

for any $z \in B\left(x_{\imath}, \varepsilon\right) \cap \mathcal{S}$. This shows $z \in B_{s}\left(P x_{\imath}, \varepsilon\right)$ for any $i=1, \cdots, N(F, \varepsilon)$, and we obtain

$$
F \subset\left\{\bigcap_{i=1}^{N(F, \varepsilon)} B\left(x_{\imath}, \varepsilon\right)\right\} \cap \mathcal{S} \subset \bigcap_{\imath=1}^{N(F, \varepsilon)} B_{s}\left(p x_{\imath}, \varepsilon\right) .
$$

Therefore we have $N_{s}(F, \varepsilon) \leqq N(F, \varepsilon)$.

Q. E. D.

Let $F$ be a relatively compact set in $\mathscr{H}, T_{1}, T_{2}$ be compact operators on $\mathscr{H}$ and $\alpha, \varepsilon_{1}, \varepsilon_{2}$ be positive numbers, then we have following inequalities.

LEMMA 2.

$$
\begin{gathered}
C(F, \varepsilon) \leqq S(F, \varepsilon), \\
S\left(\alpha T_{1}, \alpha \varepsilon_{1}\right) \leqq S\left(T_{1}, \varepsilon_{1}\right),
\end{gathered}
$$




$$
\begin{gathered}
S\left(T_{1} T_{2}, \varepsilon_{1} \varepsilon_{2}\right) \leqq S\left(T_{1}, \varepsilon_{1}\right)+S\left(T_{2}, \varepsilon_{2}\right), \\
S\left(T_{1}+T_{2}, \varepsilon_{1}+\varepsilon_{2}\right) \leqq S\left(T_{1}, \varepsilon_{1}\right)+S\left(T_{2}, \varepsilon_{2}\right) .
\end{gathered}
$$

The proof of this lemma is immediate, so we omit it.

Lemma 3. Let $\mathcal{S}$ be an $n$-dimensional Euclidean space and $\left\{\varphi_{1}, \cdots, \varphi_{n}\right\}$ be an orthonormal basis of $\mathcal{S}$. Let $T$ be the operator on $\mathcal{S}$ defined by

$$
T=\sum_{\imath=1}^{n} \lambda_{\imath}\left(\varphi_{i} \otimes \bar{\varphi}_{\imath}\right)
$$

where $\lambda_{1} \geqq \cdots \geqq \lambda_{n}>1$. Then for any $\varepsilon>0$, we have the following equality

$$
\lim _{k \rightarrow \infty} \frac{S\left(T^{k}, \varepsilon\right)}{k}=\sum_{\imath=1}^{n} \log \lambda_{\imath} .
$$

Proof. For any $k \in N$, we have

$$
T^{k}=\sum_{i=0}^{n} \lambda_{i}^{k}\left(\varphi_{\imath} \otimes \bar{\varphi}_{\imath}\right) .
$$

Now we define the following two sets:

$$
\begin{aligned}
& V_{1}=\bigcap_{i=1}^{n}\left\{y:\left|\frac{\left\langle y, \varphi_{i}\right\rangle}{\lambda_{j}^{k}}\right| \leqq \frac{1}{n^{1 / 2}}\right\}, \\
& V_{2}=\bigcap_{i=1}^{n}\left\{y:\left|\frac{\left\langle y, \varphi_{i}\right\rangle}{\lambda_{\imath}^{k}}\right| \leqq 1\right\} .
\end{aligned}
$$

Since

$$
T^{k}(U)=\left\{y: \sum_{i=1}^{n}\left|\frac{\left\langle y, \varphi_{i}\right\rangle}{\lambda_{i}^{k}}\right|^{2} \leqq 1\right\}
$$

we obtain

$$
V_{1} \subset T^{k}(U) \subset V_{2}
$$

From these formulas, we obtain

$$
C\left(V_{1}, \varepsilon\right) \leqq S\left(T^{k}, \varepsilon\right) \leqq S\left(V_{2}, \varepsilon\right) .
$$

For the upper bound of $S\left(V_{2}, \varepsilon\right)$, we construct in $V_{2}$ a cubical lattice with mesh $\varepsilon / n^{1 / 2}$ by choosing the coordinate axes $\varphi_{1}, \cdots, \varphi_{n}$. We can represent the lattice in $V_{2}$ as follows :

$$
\left\{\sum_{\imath=1}^{n} \frac{m_{i} \varepsilon}{n^{1 / 2}} \varphi_{\imath}:\left|m_{\imath}\right| \leqq\left[\frac{\lambda_{i}^{k}}{\varepsilon / n^{1 / 2}}\right], \quad m_{i} \in Z, \imath=1, \cdots, n\right\},
$$

where the symbol $[\alpha]$ means the integral part of the real number $\alpha$. According to the choice of $\varepsilon / n^{1 / 2}$, when we choose an arbitrary point $x \in V_{2}$, there is at least one point in the intersection of this lattice and $S(x, \varepsilon) . N\left(V_{2}, \varepsilon\right)$ is not 
greater than the number of such lattice points in $V_{2}$. Therefore we have

$$
S\left(V_{2}, \varepsilon\right) \leqq \sum_{i=1}^{n} \log \left\{2\left[\frac{\lambda_{2}^{k}}{\varepsilon / n^{1 / 2}}\right]+1\right\} .
$$

For the lower bound of $C\left(V_{1}, \varepsilon\right)$, we construct in $V_{1}$ a cubical lattice with mesh $2 \varepsilon$ by choosing the coordinate axes $\varphi_{1}, \cdots, \varphi_{n}$. We can represent the lattice in $V_{1}$ as follows :

$$
\left\{\sum_{\imath=1}^{n} 2 \varepsilon m_{i} \varphi_{i}:\left|m_{\imath}\right| \leqq\left[\frac{\lambda_{\imath}^{k} / n^{1 / 2}}{2 \varepsilon}\right], \quad m_{\imath} \in Z, \imath=1, \cdots, n\right\} .
$$

According to the choice of $2 \varepsilon$, the distance of any two distinct points in this lattice is not less than $2 \varepsilon . M\left(V_{1}, \varepsilon\right)$ is not less than the number of such lattice points in $V_{1}$. Therefore we obtain

$$
\sum_{i=1}^{n} \log \left\{2\left[\frac{\lambda_{\imath}^{k} / n^{1 / 2}}{2 \varepsilon}\right]+1\right\} \leqq C\left(V_{1}, \varepsilon\right) .
$$

When we note that $\lambda_{1} \geqq \cdots \geqq \lambda_{n}>1$, we obtain

$$
\lim _{k \rightarrow \infty} \frac{S\left(T^{k}, \varepsilon\right)}{k} \leqq \lim _{k \rightarrow \infty} \frac{S\left(V_{2}, \varepsilon\right)}{k} \leqq \lim _{k \rightarrow \infty} \frac{1}{k} \sum_{i=1}^{n} \log \left\{2\left[\frac{\lambda_{l}^{k}}{\varepsilon / n^{1 / 2}}\right]+1\right\}=\sum_{i=1}^{n} \log \lambda_{i} .
$$

and

$$
\lim _{k \rightarrow \infty} \frac{S\left(T^{k}, \varepsilon\right)}{k} \geqq \lim _{k \rightarrow \infty} \frac{C\left(V_{1}, \varepsilon\right)}{k} \geqq \lim _{k \rightarrow \infty} \frac{1}{k} \sum_{i=1}^{n} \log \left\{2\left[\frac{\lambda_{l}^{k} / n^{1 / 2}}{2 \varepsilon}\right]+1\right\}=\sum_{i=1}^{n} \log \lambda_{\imath} .
$$

Q.E. D.

Let $T$ be a compact positive operator on $H$ whose spectral representation is

$$
T=\sum_{i=1}^{\infty} \lambda_{i}\left(\varphi_{i} \otimes \bar{\varphi}_{\imath}\right)
$$

where $\left\{\lambda_{\imath}\right\}$ is the monotone non-increasing sequence of positive number which converges to 0 and $\left\{\varphi_{i}\right\}$ is the orthonormal system. Then we have

THEOREM 4. For any $\varepsilon>0, \alpha>0$, we have the following equality:

$$
\lim _{k \rightarrow \infty} \frac{S\left(\{\alpha T\}^{k}, \varepsilon\right)}{k}=\sum_{i=1}^{n(1 / \alpha)} \log \left(\alpha \lambda_{\imath}\right) .
$$

Proof. First we prove the case of $\alpha=1$. We define the operators $T_{1}, T_{2}$ as follows:

$$
\begin{aligned}
& T_{1}=\sum_{\imath=1}^{n(1)} \lambda_{i}\left(\varphi_{i} \otimes \bar{\varphi}_{\imath}\right), \\
& T_{2}=\sum_{\imath=n(1)+1}^{\infty} \lambda_{i}\left(\varphi_{\imath} \otimes \bar{\varphi}_{\imath}\right) .
\end{aligned}
$$

It is clear that $T=T_{1}+T_{2}$. For any $k \in N, T_{1}^{k}(U)$ is a subset of $T^{k}(U)$. There- 
fore we have

$$
N\left(T_{1}^{k}(U), \varepsilon\right)<N\left(T^{k}(U), \varepsilon\right) .
$$

From $T^{k}=T_{1}^{k}+T_{2}^{k}$, we have

$$
N\left(T^{k}(U), \varepsilon\right)=N\left(\left\{T_{1}^{k}+T_{2}^{k}\right\}(U), \varepsilon\right)
$$

From Lemma 2, we have

$$
N\left(T^{k}(U), \varepsilon\right)=N\left(\left\{T_{1}^{k}+T_{2}^{k}\right\}(U), \varepsilon\right) \leqq N\left(T_{1}^{k}(U), \varepsilon / 2\right) \cdot N\left(T_{2}^{k}(U), \varepsilon / 2\right) .
$$

As $\left\|T_{2}^{k}\right\| \leqq\left\|T_{2}\right\|^{k} \leqq 1$ holds for any $k \in N$, we get $T_{2}^{k}(U) \subset T_{2}(U)$. Therefore we have

$$
N\left(T_{2}^{k}(U), \varepsilon / 2\right) \leqq N\left(T_{2}(U), \varepsilon / 2\right) .
$$

These results imply

$$
S\left(T_{1}^{k}, \varepsilon\right) \leqq S\left(T^{k}, \varepsilon\right) \leqq S\left(T_{1}^{k}, \varepsilon / 2\right)+S\left(T_{2}, \varepsilon / 2\right) .
$$

Let $\mathcal{S}$ be a linear space spanned by $\left\{\varphi_{1}, \cdots, \varphi_{n(1)}\right\}$, i. e., an $n(1)$-dimensional subspace of $\mathscr{H}$. Since $T_{1}^{k}(U)$ is the subset of $\mathcal{S}$, we have by Lemma 1 ,

$$
S\left(T_{1}^{k}, \varepsilon\right)=S_{s}\left(T_{1}^{k}, \varepsilon\right),
$$

From this equality and Lemma 3 , we obtain

$$
\lim _{k \rightarrow \infty} \frac{S\left(T^{k}, \varepsilon\right)}{k}=\sum_{\imath=1}^{n(1)} \log \lambda_{\imath} .
$$

Next we consider the case of $\alpha>0$. By the same way as above, we decompose the compact positive operator $\alpha T$ into the following operators, say $T_{1}$ and $T_{2}$,

$$
\begin{aligned}
& T_{1}=\sum_{\imath=1}^{n(1 / \alpha)} \alpha \lambda_{i}\left(\varphi_{\imath} \otimes \bar{\varphi}_{\imath}\right), \\
& T_{2}=\sum_{\imath=n(1 / \alpha)+1}^{\infty} \alpha \lambda_{\imath}\left(\varphi_{\imath} \otimes \bar{\varphi}_{\imath}\right) .
\end{aligned}
$$

Noting next two inequalities :

$$
\begin{aligned}
& \lambda_{1} \geqq \cdots \geqq \lambda_{n(1 / \alpha)}>\frac{1}{\alpha} \geqq \lambda_{n(1 / \alpha)+1} \geqq \cdots, \\
& \alpha \lambda_{1} \geqq \cdots \geqq \alpha \lambda_{n(1 / \alpha)}>1 \geqq \alpha \lambda_{n(1 / \alpha)+1} \geqq \cdots,
\end{aligned}
$$

and applying the previous discussion to the operator $\alpha T$, we have

$$
\lim _{k \rightarrow \infty} \frac{S\left(\{\alpha T\}^{k}, \varepsilon\right)}{k}=\lim _{k \rightarrow \infty} \frac{S\left(T_{1}^{k}, \varepsilon\right)}{k}=\sum_{i=1}^{n(1 / \alpha)} \log \left(\alpha \lambda_{\imath}\right) .
$$

Q.E. D.

Let $\{\xi(t): 0 \leqq t \leqq 1\}$ be a mean continuous real Gaussian process. We denote 
by $K(\cdot, \cdot)$ the covariance function of $\xi$, i. e.,

$$
K(s, t)=E\{\xi(s)-E \xi(s)\}\{\xi(t)-E \xi(t)\},
$$

where $s, t \in[0,1]$ and the letter $E$ denotes the expectation values of random variables. We define the integral kernel operator $T$ on $L^{2}[0,1]$ by

$$
(T f)(t)=\int_{0}^{1} K(s, t) f(s) d s,
$$

where $f \in L^{2}[0,1], 0 \leqq t \leqq 1$. Because of the mean continuity of $\xi, K(\cdot, \cdot)$ is jointly continuous on $[0,1]^{2}$. Therefore, by Mercer's theorem (Ash [1], Umegaki-Ohya [15]), $K(\cdot, \cdot)$ can be represented as follows :

$$
K(s, t)=\sum_{\imath=1}^{\infty} \lambda_{\imath} \varphi_{\imath}(s) \varphi_{i}(t),
$$

where $s, t \in[0,1]$ and $\left\{\lambda_{2}\right\}$ is a monotone non-increasing sequence which converges to 0 and $\left\{\varphi_{i}\right\}$ is an orthonormal system. The summation of the right hand side is in the sense of the uniform convergence on $[0,1]^{2}$. We note that the spectral representation of $T$ can be denoted as

$$
T=\sum_{i=1}^{\infty} \lambda_{i}\left(\varphi_{i} \otimes \varphi_{i}\right)
$$

We define a function in order to explain Pinsker's theorem. Let $\varepsilon$ be in $\left[0,\left\{\sum_{\imath=1}^{\infty} \lambda_{\imath}\right\}^{1 / 2}\right]$ and $\vartheta$ be a non-negative number determined by the following equality :

$$
\varepsilon^{2}=\sum_{\imath=1}^{\infty} \min \left[\lambda_{\imath}, \vartheta\right]
$$

We denote the function from $\varepsilon$ to $\vartheta$ by $f$, i. e., $\vartheta=f(\varepsilon)$. It is known that $f(\varepsilon)$ is a monotone increasing and continuous function defined on $\left[0,\left\{\sum_{\imath=1}^{\infty} \lambda_{\imath}\right\}^{1 / 2}\right]$ (Baba-Kaji-Ihara [2]). The range of $f$ is $\left[0, \lambda_{1}\right]$. We denote by $g$ the inverse function of $f$ which is defined on $\left[0, \lambda_{1}\right]$. We can represent this function $g$ explicitly, i. e.,

$$
\varepsilon=g(\vartheta)=\left\{n(\vartheta) \vartheta+\sum_{\imath=n(\theta)+1}^{\infty} \lambda_{\imath}\right\}^{1 / 2}
$$

Now we can explain Pinsker's theorem.

THEOREM 5 (Pinsker [7]).

$$
H(\xi, \varepsilon)=\frac{1}{2} \sum_{i=1}^{\infty} \log \max \left[\frac{\lambda_{\imath}}{f(\varepsilon)}, 1\right] .
$$

By using the above theorem, we have next theorem.

THEOREM 6. Let $\{\boldsymbol{\xi}(t): 0 \leqq t \leqq 1\}$ be a mean contrnnous Gaussian process, $K(\cdot, \cdot)$ be the covariance function of $\xi$ and $T$ be the integral kernel operator 
induced by $K(\cdot, \cdot)$ on $L^{2}[0,1]$. Then, for any $a>0$, we have the following equality.

$$
\lim _{k \rightarrow \infty} \frac{S\left(T^{k}, a \cdot f(\varepsilon)^{k}\right)}{k}=2 H(\xi, \varepsilon) .
$$

Proof. By Theorem 4, we have

$$
\lim _{k \rightarrow \infty} \frac{S\left(T^{k}, a \cdot f(\varepsilon)^{k}\right)}{k}=\lim _{k \rightarrow \infty} \frac{S\left(\{T / f(\varepsilon)\}^{k}, a\right)}{\dot{k}}=\sum_{i=1}^{n(f(\varepsilon))} \log \left(\frac{\lambda_{2}}{f(\varepsilon)}\right) .
$$

Now, we have by Theorem 5 ,

$$
2 H(\xi, \varepsilon)=\sum_{i=1}^{\infty} \log \max \left[\frac{\lambda_{\imath}}{f(\varepsilon)}, 1\right]=\sum_{\imath=1}^{n(f(\varepsilon))} \log \left(\frac{\lambda_{\imath}}{f(\varepsilon)}\right) .
$$

Q. E. D.

\section{Orders of growth of $\varepsilon$-entropy in Gaussian processes.}

In this section, we see the asymptotic behavior of $H(\xi, \varepsilon)$ when $\varepsilon$ is suffciently small. Let $\{\xi(t): 0 \leqq t \leqq 1\}$ be a mean continuous Gaussian process whose covariance function is $K(\cdot, \cdot)$, and $T$ be the integral kernel operator on $L^{2}[0,1]$ induced by $K(\cdot, \cdot)$. According to Prosser [9], we define the order of growth $\rho$ of $S(T, \varepsilon)$ as follows :

$$
\rho=\varlimsup_{\varepsilon \rightarrow+0} \frac{\log S(T, \varepsilon)}{\log (1 / \varepsilon)} .
$$

Then we have the following theorem.

Theorem 7 (Levin [6], Prosser [9]).

$$
\rho=\varlimsup_{\varepsilon \rightarrow+0} \frac{\log n(\varepsilon)}{\log 1 / \varepsilon}=\inf \left\{\nu>0: \sum_{k=1}^{\infty} \lambda_{k}^{\nu}<\infty\right\},
$$

where $\left\{\lambda_{k}\right\}$ is the monotone non-increasing sequence of proper values of $T$. result.

When we discuss the order of growth of $H(\xi, \varepsilon)$, we get the following

THEOREM 8.

$$
\frac{2 \rho}{1-\rho} \geqq \lim _{\varepsilon \rightarrow+0} \frac{\log H(\xi, \varepsilon)}{\log (1 / \varepsilon)}
$$

Proof. From Lemma 2, we have

$$
S\left(T^{k}, \varepsilon^{k}\right) \leqq k S(T, \varepsilon), \quad k \in N .
$$

Taking $a=1$ in Theorem 6 , we get

$$
S(T, f(\varepsilon)) \geqq \lim _{k \rightarrow+\infty} \frac{\log S\left(T^{k}, f(\varepsilon)^{k}\right)}{k}=2 H(\xi, \varepsilon) .
$$

From this we have $S(T, \varepsilon) \geqq 2 H(\xi, g(\varepsilon))$. 
Then we obtain the following inequalities:

$$
\begin{aligned}
& \frac{\log 2 H(\xi, g(\varepsilon))}{\log \{1 / g(\varepsilon)\}} \leqq \frac{\log S(T, \varepsilon)}{\log (1 / \varepsilon)} \cdot \frac{\log \varepsilon}{\log g(\varepsilon)} \\
& =\frac{\log S(T, \varepsilon)}{\log (1 / \varepsilon)} \cdot \frac{\log \varepsilon}{\log \left\{n(\varepsilon) \varepsilon+\sum_{k=n(\varepsilon)+1}^{\infty} \lambda_{k}\right\}^{1 / 2}} \\
& \leqq \frac{\log S(T, \varepsilon)}{\log (1 / \varepsilon)} \cdot \frac{2}{1-\{\log n(\varepsilon) / \log (1 / \varepsilon)\}}
\end{aligned}
$$

Since $g(\varepsilon)$ converges to 0 as $\varepsilon$ converges to 0 , we have

$$
\varlimsup_{\varepsilon \rightarrow+0} \frac{\log 2 H(\xi, g(\varepsilon))}{\log 1 / g(\varepsilon)} \leqq \frac{2 \rho}{1-\rho},
$$

by Theorem 7 .

Q.E. D.

Remark. When $T$ is the integral kernel operator induced by a mean continuous Gaussian process $\{\xi(t): 0 \leqq t \leqq 1\}, T$ is a trace class operator on $L^{2}[0,1]$. Therefore we can get $\rho \leqq 1$. Especially if we treat the Brownian motion $\{B(t): 0 \leqq t \leqq 1\}$ (Umegaki-Ohya [15]), then $\rho=1 / 2$. Therefore we have the following inequality:

$$
\varlimsup_{\varepsilon \rightarrow+0} \frac{\log H(B, \varepsilon)}{\log (1 / \varepsilon)} \leqq 2 \text {. }
$$

Acknowledgement. The author would like to express his hearty thanks to Professor Hisaharu Umegaki and Professor Masanori Ohya for their valuable suggestions and encouragement in the course of preparing this paper.

\section{REFERENCES}

[1] R.B. AsH, Information Theory, John Wiley, 1965.

[2] Y. BABA, K. Kaji ANd S. Ihara, $\varepsilon$-Entropy of Gaussian Processes, Seminar on Probability, Vol. 29 (1968), (in Japanese).

[3] T. Hida ANd M. Hitsuda, Gaussian Processes, Kinokuniya, 1976, (in Japanese).

[4] S. IHARA, Stochastic Processes and Entropies, Iwanami, 1984, (in Japanese).

[5] A. N. Kolmogorov, Theory of transmission of information, Amer. Math. Soc. Transl. Ser. 2, Vol. 33 (1963), 291-321.

[6] B. Ja. Levin, Distribution of Zeros of Entire Functions, Amer. Math. Soc. Monogr. Transl., Vol. 5 (1964).

[7] M.S. Pinsker, Gaussian sources, Problemy Peredači Informaci, Vol. 14 (1963), 59-100, (in Russian).

[8] M.S. PINSKER, Information and Information Stability of Random Variables and Processes, Holden-Day, 1964.

[9] R.T. Prosser, The $\varepsilon$-entropy and $\varepsilon$-capacity of certain time-varing channels, J. Math. Anal. Appl., Vol. 16 (1966), 365-398. 
[10] R. Schatten, Norm Ideals of Complete Continuous Operators, Springer Verlag, 1970.

[11] H. UMEGAKI, Conditional expectation in an operator algebra (entropy and information), Kodai Math. Sem. Rep., Vol. 14 (1962), 59-85.

[12] H. UmegaKi, A functional method on amount of entropy, Kodai Math. Sem. Rep., Vol. 15 (1963), 162-175.

[13] H. UMEGAKI, General treatment of alphabet-message space and integral representation of entropy, Kodai Math. Sem. Rep., Vol. 16 (1964), 18-26.

[14] H. UMEgAKI, A functional method for stationary channels, Kodai Math. Sem. Rep., Vol. 16 (1964), 27-39.

[15] H. Umegaki And M. OHya, Entropies in Probability Theory, Kyoritsu, 1983, (in Japanese).

[16] H. UMEGAKI AND M. OHYA, Quantum Mechanical Entropies, Kyoritsu, 1984, (in Japanese).

[17] H. Umegaki, M. Ohya and F. Hiai, Introduction to Operator Algebras, Kyoritsu, 1985, (in Japanese).

Department of Information SCIENCES

TOKyo Institute of TeChNology

Oh-okayama, Meguro-ku, Tokyo, Japan 